\title{
Exercise capacity impairment after COVID-19 pneumonia is mainly caused by deconditioning
}

\begin{abstract}
To the Editor:
Copyright (The authors 2022

This version is distributed under the terms of the Creative Commons Attribution Non-Commercial Licence 4.0. For commercial reproduction rights and permissions contact permissions@ersnet.org

Received: 23 April 2021 Accepted: 10 Oct 2021

\section{๑@®@}

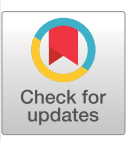

The new severe acute respiratory syndrome (SARS) coronavirus 2 (SARS-CoV-2) can cause severe pneumonia characterised by dry cough, dyspnoea, hypoxaemia and diffuse ground glass opacities on chest computed tomography (CT) [1]. While much has been learnt concerning diagnosis and treatment of coronavirus disease 2019 (COVID-19) during the first year of the pandemic, only scarce data is available concerning post-COVID long-term pulmonary sequelae. Data about pulmonary function in early convalescence demonstrated impaired diffusion capacity, lower respiratory muscle strength, and radiological abnormalities [2, 3]. Herein we report data of cardio-pulmonary exercise testing (CPET) 3 months after severe COVID-19 pneumonitis.

Between 26 February and 3 May, 2020, 221 patients with RT-PCR-confirmed SARS-CoV-2 infection were admitted to the University Hospital Basel, Switzerland. A total of 50 (22.6\%) patients suffered from severe COVID-19 pneumonitis, fulfilling $\geqslant 2$ of the following criteria: respiratory rate $>30$ breaths $\cdot \mathrm{min}^{-1}$, peripheral oxygen saturation $\left(S_{\mathrm{pO}_{2}}\right)<93 \%$ while breathing ambient air, C-reactive protein (CRP) levels $>75 \mathrm{mg} \cdot \mathrm{L}^{-1}$ (normal $<10.0 \mathrm{mg} \cdot \mathrm{L}^{-1}$ ), ground glass opacities or diffuse infiltrates on $\mathrm{CT}$ scan, or progression of CT findings $>50 \%$ within $24-48$ h or typical CT findings $\geqslant 4$ lobes [4]. Patients were treated according to local standards at that time, and only one patient received systemic corticosteroids (cumulative dose of $80 \mathrm{mg}$ prednisone); all patients were included in an observational study (NCT04351503).

During hospitalisation 5/50 (10\%) patients died due to COVID-19. One further patient succumbed to pre-existing haematological disease after hospital discharge. Among the 44 COVID-19 survivors, four experienced prolonged hospitalisation with oxygen dependency. A further five patients declined to undertake CPET. All patients received physiotherapy during their hospitalisation; after discharge, 8/35 (23\%) patients were transferred for further inpatient pulmonary rehabilitation, and 3/35 (9\%) patients underwent outpatient pulmonary rehabilitation. In all these patients, pulmonary rehabilitation programmes were completed at the time of CPET.

In the context of this analysis, patients were followed-up, including clinical status, body plethysmography and chest CT scan 3 months after COVID-19 pneumonitis.

All 35 patients underwent an incremental CPET using a cycle ergometer [5] (Ergoline Ergoselect 1000) in semi-recumbent position in continuous ramp mode $\left(10-20 \mathrm{~W} \cdot \mathrm{min}^{-1}\right)$. CPET parameters were systematically computed according to breath-by-breath analysis and data were displayed online (Sentry Suite Version 3.10). Arterial blood gas analysis at rest and at maximal exercise level was performed (ABL 800 Flex). The level of dyspnoea and exhaustion at peak exercise was objectified using the Borg modified scale [6]. The evaluation of the collected data was in accordance with the Wassermann algorithm [7] and the adaptation of Schmid et al. [8]. Health-related quality of life (QoL) was evaluated using the St George's Respiratory Questionnaire (SGRQ) and the King’s Brief Interstitial Lung Disease (K-BILD) questionnaire. Chest CT scans were conducted on a dual source CT scanner (Somatom Definition Flash, Siemens Healthineers) using dual-energy acquisition after intravenous injection of iodine contrast material

Shareable abstract (@ERSpublications)

Not pulmonary factors, but physical deconditioning is the main limiting factor of exercise capacity in patients after severe COVID-19 pneumonitis. This underscores the importance of an early rehabilitative intervention in these patients. https://bit.ly/2XVvr6C

Cite this article as: Jahn K, Sava M, Sommer G, et al. Exercise capacity impairment after COVID-19 pneumonia is mainly caused by deconditioning. Eur Respir J 2022; 59: 2101136 [DOI: 10.1183/ 13993003.01136-2021].
\end{abstract}


in the late pulmonary arterial phase. Mann-Whitney U-test was computed to assess statistical differences; a p-value $<0.05$ was considered statistically significant.

Baseline characteristics, underlying comorbidities, and concomitant medication of those 35 patients who agreed to perform CPET are shown in table 1. On chest CT scan, 15/35 patients (43\%) exhibited residuals only, and 6/35 patients (17\%) had additional fibrotic changes. Pulmonary function values were normal (total lung capacity $\geqslant 80 \%$ predicted, diffusion capacity of the lung for carbon monoxide $\left(D_{\text {LCO }}\right) \geqslant 80 \%$ predicted, Tiffeneau index $>0.7$ ) in $23 / 35$ patients $(66 \%)$. A normal maximal oxygen uptake $\left(V_{\mathrm{O}_{2} \max }\right)$ during CPET ( $V_{\mathrm{O}_{2} \max } \geqslant 82 \%$ predicted) was observed in $16 / 35$ (46\%) patients; $19 / 35$ (54\%) proved to have impaired $V_{\mathrm{O}_{2} \max }$ (15 with mild impairment: $V_{\mathrm{O}_{2} \max } 61-81 \%$ predicted; four with moderate impairment: $V_{\mathrm{O}_{2} \max } 51-60 \%$ predicted). The main limiting factors in those patients with impaired $V_{\mathrm{O}_{2} \max }$ were deconditioning in $9 / 19$, cardiovascular in $5 / 19$, and pulmonary limitations in 5/19 patients. In those patients with impaired $V_{\mathrm{O}_{2} \text { max }}, D_{\mathrm{LCO}} \%$ pred at day 90 was significantly lower as compared to the patients with normal $V_{\mathrm{O}_{2} \max }(\mathrm{p}=0.006)$; no other parameter differed between the two groups (table 1). Detailed variables of CPET are shown in table 1.

Contrary to our expectations, both in patients with and without lung function impairment, the most common main limiting factor of $V_{\mathrm{O}_{2} \max }$ was not of a pulmonary nature, but general deconditioning. Of note, maximal inspiratory pressure (MIP) and maximal expiratory pressure (MEP) were normal in those patients with deconditioning (mean MIP 99.4\% predicted, mean MEP 79.9\% predicted), making it unlikely that neuromuscular impairment caused the limitation. Three of the nine patients with deconditioning were obese. In $14 \%$ of the patients (5/35), cardiovascular limitation of CPET was observed. From those, three had previous cardiovascular disease. Only one of the five patients with pulmonary limitation had a pre-existing respiratory disease, i.e. severe obstructive sleep apnoea. Health-related QoL was not better in those with normal $V_{\mathrm{O}_{2} \max }$ compared to those with impaired $V_{\mathrm{O}_{2} \max }$. However, this finding is limited by the fact that $40 \%$ of patients did not complete the questionnaires, though these were equally distributed in both groups (42\% versus $37.5 \%)$.

Our data on pulmonary function impairment after COVID-19 pneumonitis are in line with previous publications [2, 9-11]. The low prevalence of respiratory comorbidities and active smoking in our population might partially explain the finding of $66 \%$ normal pulmonary function tests 3 months after severe COVID-19. Impairment of physical performance in patients recovering from COVID-19 pneumonia has been described before [2, 3, 10, 12]. To date, there are accumulating data on CPET after SARS-CoV-2 infection [13-18], but only limited respective information on patients surviving severe COVID-19 pneumonitis. In our well-characterised patient group, almost half (46\%) had normal $V_{\mathrm{O}_{2} \max }$ on CPET. In those patients with impaired $V_{\mathrm{O}_{2} \max }$, the majority (47\%) was limited by deconditioning. Thus, despite the severity of the acute COVID-19 pneumonitis, only 14\% of survivors exhibited pulmonary limitation in CPET 3 months later, demonstrating a surprisingly good pulmonary recovery. This applies even more in the light of the predominant lung pathology observed in patients with severe COVID-19, i.e. diffuse alveolar damage, endothelitis and pulmonary immunothrombosis [19].

In a previous issue of the European Respiratory Journal, SKJøRTEN et al. [15] reported a reduced $V_{\mathrm{O}_{2} \max }$ in one-third of COVID-19 patients 3 months after hospital discharge, with deconditioning being the major cause of exercise limitation. Compared to these findings, we observed a higher proportion of patients with impaired peak oxygen uptake (54\%), which is likely due to the more severe disease course in our patient group, as depicted by the longer length of hospital stay (median 14 day versus median 6 days) [15]. The study by RINALDo et al. [13] reported CPET data in a mixed group of patients with critical, severe and mild-moderate COVID-19. Even though we included only patients with severe COVID-19 pneumonia, the mean $V_{\mathrm{O}_{2} \text { max }}$ of $82 \%$ predicted in our study population is surprisingly similar to the one reported by RinALDo et al. [13] (83\%). A striking disparity between the two patient groups is the portion of active smokers, being only $2.9 \%$ in our population, as compared to $19 \%$ in the group studied by RinALDo et al. [13]. This might, at least partially, explain the good outcome in our patients. Finally, in line with our data, RINALDo et al. [13] found muscle deconditioning to be the main cause of reduced exercise capacity [13]. In a very small group of 10 moderate and severe COVID-19 patients, GAO et al. [20] performed CPET 1 month post-discharge. In contrast to our data, GAO et al. [20] found reduced peak oxygen uptake in all cases, an apparent contradiction which might be explained by the 2 months later follow-up time-point of our study. However, similar to us, GAO et al. [20] showed that extrapulmonary factors were the main reason for exercise limitation. Notably, our findings are in line with CPET data from survivors of the 2002 coronavirus-induced SARS outbreak: 3 months after SARS, $41 \%$ of the patients showed reduced $V_{\mathrm{O}_{2} \max }$; none of these patients had pulmonary limitation, but extrapulmonary disease, mainly impaired muscle function, caused reduced $V_{\mathrm{O}_{2} \max }$ [21]. Furthermore, similar data were found in survivors of severe acute 
TABLE 1 Baseline characteristics, underlying comorbidities, concomitant medication, disease severity at hospital admission, pulmonary function, radiological outcome, quality of life and cardiopulmonary exercise testing data 3 months after severe COVID-19 pneumonitis

\begin{tabular}{|c|c|c|c|c|}
\hline & Total & $\begin{array}{c}\text { Impaired } \\
V_{\mathrm{O}_{2} \max }\end{array}$ & $\begin{array}{l}\text { Normal } \\
V_{\mathrm{O}_{2} \max } \#\end{array}$ & p-value \\
\hline Patients, $\mathrm{n}$ & 35 & 19 & 16 & \\
\hline Age, years & $58 \pm 13$ & $56 \pm 13$ & $60 \pm 14$ & 0.289 \\
\hline Ethnicity & & & & 0.232 \\
\hline Caucasian & $23(65.7)$ & $11(57.9)$ & $12(75.0)$ & \\
\hline Asian & $9(25.7)$ & $5(26.3)$ & $4(25.0)$ & \\
\hline African & $3(8.6)$ & $3(15.8)$ & 0 & \\
\hline Female sex & $6(17.1)$ & $2(10.5)$ & $4(25.0)$ & 0.379 \\
\hline \multicolumn{5}{|l|}{ Comorbidities } \\
\hline Hypertension & $15(42.9)$ & $7(36.8)$ & $8(50.0)$ & 0.506 \\
\hline Diabetes & $7(20.0)$ & $5(26.3)$ & $2(12.5)$ & 0.415 \\
\hline Asthma & $3(8.6)$ & $2(10.5)$ & $1(6.2)$ & 1 \\
\hline Coronary heart disease & $2(5.7)$ & $1(5.3)$ & $1(6.2)$ & 1 \\
\hline COPD & 0 & 0 & 0 & \\
\hline Chronic kidney disease & $3(8.6)$ & $2(10.5)$ & $1(6.2)$ & 1 \\
\hline Autoimmune disease & 2 & 0 & 2 & 0.202 \\
\hline HIV & $2(5.7)$ & $2(10.5)$ & 0 & 0.489 \\
\hline $\mathrm{BMI}, \mathrm{kg} \cdot \mathrm{m}^{-2}$ & $29 \pm 5$ & $28 \pm 3$ & $31 \pm 6$ & 0.2 \\
\hline Smoking & & & & 0.517 \\
\hline Active smoker & $1(2.9)$ & 0 & $1(6.2)$ & \\
\hline Former smoker & $10(28.6)$ & $6(31.6)$ & $4(25.0)$ & \\
\hline Never smoker & $24(68.6)$ & $13(68.4)$ & $11(68.8)$ & \\
\hline Any concomitant medication & $20(57.1)$ & $9(47.4)$ & $11(68.8)$ & 0.306 \\
\hline ACE inhibitor & $9(25.7)$ & $5(26.3)$ & $4(25.0)$ & 1 \\
\hline Statins & $5(14.3)$ & $2(10.5)$ & $3(18.8)$ & 0.642 \\
\hline Antidiabetic drugs & $8(22.9)$ & $6(31.6)$ & $2(12.5)$ & 0.244 \\
\hline Oral anticoagulation & 0 & 0 & 0 & \\
\hline WHO scale at hospitalisation & & & & 0.745 \\
\hline 3: hospitalised, no oxygen therapy & $18(51.4)$ & $10(52.6)$ & $8(50.0)$ & \\
\hline $\begin{array}{l}4 \text { or 5: hospitalised, oxygen by mask or nasal prongs; noninvasive ventilation or high-flow } \\
\text { oxygen }\end{array}$ & $14(40.0)$ & $8(42.1)$ & $6(37.5)$ & \\
\hline $\begin{array}{l}6 \text { or 7: hospitalised, intubation and mechanical ventilation; ventilation plus additional } \\
\text { organ support }\end{array}$ & $3(8.6)$ & $1(5.3)$ & $2(12.5)$ & \\
\hline CRP peak value during hospitalisation, $\mathrm{mg} \cdot \mathrm{L}^{-1}$, median (IQR) & $72(29,121)$ & $68.1(27,121)$ & $74.2(37,142)$ & 0.854 \\
\hline Hospital length of stay, days, median (IQR) & $14(9,24)$ & $11(8,28)$ & $14.5(10,21)$ & 0.854 \\
\hline \multicolumn{5}{|l|}{ Pulmonary rehabilitation after hospital discharge } \\
\hline Inpatient pulmonary rehabilitation after discharge & $8(23)$ & $5(14)$ & $3(9)$ & 0.7 \\
\hline Outpatient pulmonary rehabilitation after discharge & $3(9)$ & $2(6)$ & $1(3)$ & 1 \\
\hline \multicolumn{5}{|l|}{ Pulmonary function tests day 90} \\
\hline TLC \% pred & $93 \pm 9$ & $91 \pm 9$ & $96 \pm 9$ & 0.153 \\
\hline FVC $\%$ pred & $91 \pm 13$ & $88 \pm 12$ & $94 \pm 14$ & 0.119 \\
\hline $\mathrm{FEV}_{1} \%$ pred & $93 \pm 13$ & $90 \pm 11$ & $96 \pm 15$ & 0.226 \\
\hline $\mathrm{FEV}_{1} / \mathrm{FVC} \%$ & $83 \pm 10$ & $84 \pm 12$ & $82 \pm 9$ & 0.703 \\
\hline$D_{\mathrm{Lco}} \%$ pred & $88 \pm 17$ & $80 \pm 13$ & $96 \pm 18$ & 0.006 \\
\hline \multicolumn{5}{|l|}{ Radiological findings day $90^{\S}$} \\
\hline Residuals & $21(61.8)$ & $11(57.9)$ & $10(66.7)$ & 0.728 \\
\hline Fibrosis & $6(17.6)$ & $2(10.5)$ & $4(26.7)$ & 0.37 \\
\hline Embolism & 0 & 0 & 0 & \\
\hline Disturbed microcirculation & $11(34.4)$ & $6(31.6)$ & $5(38.5)$ & 0.721 \\
\hline \multicolumn{5}{|l|}{ Health related quality of life day 90} \\
\hline SGRQ completed" & $21(60)$ & $11(31)$ & $10(29)$ & 1 \\
\hline SGRQ total score, median (IQR) & $13(5,27)$ & $10(6,20)$ & $22(8,30)$ & 0.359 \\
\hline SGRQ symptoms, median (IQR) & $12(0,21)$ & $9(2,16)$ & $17(2,29)$ & 0.285 \\
\hline SGRQ activity, median (IQR) & $30(11,48)$ & $18(6,38)$ & $39(22,52)$ & 0.242 \\
\hline SGRQ impact, median (IQR) & $6(4,15)$ & $4(3,12)$ & $14(5,17)$ & 0.357 \\
\hline K-BILD completed ${ }^{+}$ & $21(60)$ & $11(31)$ & $10(29)$ & 1 \\
\hline K-BILD total score, median (IQR) & $87(72,93)$ & $89(73,92)$ & $82(72,93)$ & 0.75 \\
\hline K-BILD breathlessness/activity, median (IQR) & $85(62,100)$ & $89(72,98)$ & $78(64,99)$ & 0.668 \\
\hline K-BILD psychological, median (IQR) & $80(72,88)$ & $80(64,88)$ & $78(75,87)$ & 0.859 \\
\hline K-BILD chest symptoms, median (IQR) & $91(69,100)$ & $91(69,100)$ & $91(62,100)$ & 0.941 \\
\hline
\end{tabular}


TABLE 1 Continued

\begin{tabular}{|c|c|c|c|c|}
\hline & Total & $\begin{array}{c}\text { Impaired } \\
V_{\mathrm{O}_{2} \max }\end{array}$ & $\begin{array}{l}\text { Normal } \\
V_{\mathrm{O}_{2} \max } \#\end{array}$ & $\mathrm{p}$-value \\
\hline \multicolumn{5}{|l|}{ Cardiopulmonary exercise testing day 90} \\
\hline Workload \% pred & $97 \pm 22$ & $85 \pm 18$ & $110 \pm 18$ & 0.001 \\
\hline$V_{\mathrm{O}_{2}} \max \%$ pred & $82 \pm 16$ & $71 \pm 9$ & $96 \pm 10$ & $<0.001$ \\
\hline \multicolumn{5}{|l|}{ Circulation } \\
\hline Heart rate at rest, bpm & $74 \pm 14$ & $72 \pm 14$ & $76 \pm 14$ & 0.371 \\
\hline Heart rate at peak, bpm & $129 \pm 30$ & $126 \pm 37$ & $132 \pm 18$ & 0.529 \\
\hline Heart rate reserve at peak, $L \cdot \mathrm{min}^{-1}$ & $34 \pm 20$ & $37 \pm 21$ & $29 \pm 19$ & 0.267 \\
\hline $\mathrm{O}_{2}$ pulse at rest, $\mathrm{mL}$ & $7 \pm 7$ & $6 \pm 2$ & $7 \pm 10$ & 0.064 \\
\hline $\mathrm{O}_{2}$ pulse at peak, $\mathrm{mL}$ & $14 \pm 2$ & $13 \pm 2$ & $15 \pm 2$ & 0.022 \\
\hline $\mathrm{O}_{2}$ pulse at peak, $\%$ pred & $103 \pm 21$ & $91 \pm 13$ & $118 \pm 20$ & $<0.001$ \\
\hline $\mathrm{SBP}$ at rest, $\mathrm{mmHg}$ & $135 \pm 18$ & $135 \pm 18$ & $135 \pm 19$ & 0.934 \\
\hline SBP at peak, $\mathrm{mmHg}$ & $166 \pm 31$ & $161 \pm 32$ & $172 \pm 31$ & 0.214 \\
\hline $\mathrm{DBP}$ at rest, $\mathrm{mmHg}$ & $83 \pm 12$ & $84 \pm 12$ & $83 \pm 12$ & 0.829 \\
\hline DBP at peak, $\mathrm{mmHg}$ & $93 \pm 18$ & $90 \pm 14$ & $96 \pm 22$ & 0.54 \\
\hline \multicolumn{5}{|l|}{ Ventilation } \\
\hline Minute ventilation at rest, $L \cdot \mathrm{min}^{-1}$ & $13 \pm 6$ & $14 \pm 6$ & $12 \pm 5$ & 0.319 \\
\hline Minute ventilation at peak, $L \cdot \mathrm{min}^{-1}$ & $66 \pm 20$ & $66 \pm 24$ & $67 \pm 15$ & 0.573 \\
\hline Minute ventilation at peak, $\%$ pred & $73 \pm 14$ & $68 \pm 15$ & $79 \pm 10$ & 0.018 \\
\hline Breathing rate at rest, $L \cdot \min ^{-1}$ & $19 \pm 9$ & $18 \pm 9$ & $21 \pm 8$ & 0.233 \\
\hline Breathing rate at peak, L. $\min ^{-1}$ & $37 \pm 8$ & $36 \pm 9$ & $38 \pm 8$ & 0.446 \\
\hline Breathing reserve at peak, \% & $39 \pm 15$ & $43 \pm 16$ & $34 \pm 13$ & 0.116 \\
\hline \multicolumn{5}{|l|}{ Gas exchange } \\
\hline $\mathrm{O}_{2}$ saturation at rest, $\%$ & $97 \pm 2$ & $98 \pm 2$ & $97 \pm 2$ & 0.085 \\
\hline $\mathrm{O}_{2}$ saturation at peak, $\%$ & $97 \pm 2$ & $97 \pm 1$ & $97 \pm 2$ & 0.859 \\
\hline $\mathrm{P}_{\mathrm{aO}_{2}}$ at rest, $\mathrm{kPa}$ & $11 \pm 2$ & $11 \pm 2$ & $11 \pm 2$ & 0.987 \\
\hline$P_{\mathrm{aO}_{2}}$ at peak, $\mathrm{kPa}$ & $14 \pm 1$ & $14 \pm 1$ & $14 \pm 1$ & 0.986 \\
\hline$P_{\mathrm{aCO}_{2}}$ at rest, $\mathrm{kPa}$ & $5 \pm 0.5$ & $5 \pm 0.6$ & $5 \pm 0.4$ & 0.842 \\
\hline$P_{\mathrm{aCO}_{2}}$ at peak, $\mathrm{kPa}$ & $4.9 \pm 0.5$ & $4.9 \pm 0.6$ & $5 \pm 0.5$ & 0.743 \\
\hline Lactate at rest, $\mathrm{mmol} \cdot \mathrm{L}^{-1}$ & $1.4 \pm 0.6$ & $1.4 \pm 0.6$ & $1.5 \pm 0.6$ & 0.475 \\
\hline Lactate at peak, $\mathrm{mmol} \cdot \mathrm{L}^{-1}$ & $7 \pm 3$ & $7 \pm 3$ & $7 \pm 2$ & 0.945 \\
\hline Borg dyspnea & $5 \pm 2$ & $4 \pm 2$ & $5 \pm 2$ & 0.181 \\
\hline Borg peripheral limitation & $5 \pm 2$ & $6 \pm 3$ & $5 \pm 2$ & 0.634 \\
\hline Workload limitation & & & & 0.061 \\
\hline No limitation & $28(80.0)$ & $12(63.2)$ & $16(100)$ & \\
\hline Mild limitation & $4(11.4)$ & $4(21.1)$ & 0 & \\
\hline Moderate limitation & $2(5.7)$ & $2(10.5)$ & 0 & \\
\hline Severe limitation & $1(2.9)$ & $1(5.3)$ & 0 & \\
\hline$V_{\mathrm{O}_{2}} \max$ limitation & & & & $<0.001$ \\
\hline No limitation & $16(45.7)$ & 0 & $16(100)$ & \\
\hline Mild limitation & $15(42.9)$ & $15(78.9)$ & 0 & \\
\hline Moderate limitation & $4(11.4)$ & $4(21.1)$ & 0 & \\
\hline Pulmonary gas exchange & & & & 0.215 \\
\hline Drop in $P_{\mathrm{aO}_{2}}$ & $2(5.7)$ & 0 & $2(12.5)$ & \\
\hline Increase in $P_{\mathrm{aCO}}$ & $5(14.3)$ & $3(15.8)$ & $2(12.5)$ & \\
\hline Desaturation & $3(8.6)$ & $2(10.5)$ & $1(6.2)$ & 1 \\
\hline Any cardiac limitations & $19(54.3)$ & $10(52.6)$ & $9(56.3)$ & 1 \\
\hline Main limiting factor & & & & $<0.0001$ \\
\hline Cardiovascular & $5(14.3)$ & $5(26.3)$ & 0 & \\
\hline Deconditioning & $9(25.7)$ & $9(47.4)$ & 0 & \\
\hline Pulmonary & $5(14.3)$ & $5(26.3)$ & 0 & \\
\hline No limitation & $16(45.7)$ & 0 & $16(100)$ & \\
\hline
\end{tabular}

Data are presented as $\mathrm{n}(\%)$ or mean \pm SD, unless otherwise indicated. ACE: angiotensin-converting enzyme; BMI: body mass index; bpm: beats per minute; CRP: C-reactive protein; DBP: diastolic blood pressure; $D_{\mathrm{LCo}}$ : diffusing capacity of the lung for carbon monoxide; FEV ${ }_{1}$ : forced expiratory volume in $1 \mathrm{~s}$; FVC: forced vital capacity; IQR: interquartile range; K-BILD: King's Brief Interstitial Lung Disease questionnaire; $P_{\text {aO }}$ : arterial oxygen tension; $P_{\mathrm{acO}_{2}}$ : arterial carbon dioxide tension; SBP: systolic blood pressure; SGRQ: St George's Respiratory Questionnaire; TLC: total lung capacity; $V_{\mathrm{O}_{2} \max }$ : maximal oxygen uptake; WHO: World Health Organization. \#: normal $V_{\mathrm{O}_{2}}$ max is defined as $V_{\mathrm{O}_{2}}$ max $\geqslant 82 \%$ predicted; mild impairment as $61-81 \%$ predicted, moderate impairment as $51-60 \%$ predicted. ": the SGRQ is a 50 -item questionnaire divided into three domains (symptoms, activity, impact); a total and three domain scores are calculated, each domain is scored separately; a score of zero represents best, a value of 100 represents worst quality of life. ${ }^{+}$: the K-BILD questionnaire is a 15-item questionnaire divided into three domains (breathlessness and activity, psychological aspects, chest symptoms); a score of 100 represents best, a value of zero represents worst quality of life. ${ }^{\S}$ : ground glass opacities and reticulations were considered as residuals, coarse reticulations and traction bronchiectasis were interpreted as fibrosis; microcirculation was assessed based on dual energy computed tomography. 
respiratory distress syndrome (ARDS) caused by various aetiologies [22]. By performing CPET 3 months after severe COVID-19, a time point by which most radiological abnormalities related to the acute infection had vanished, we were able to demonstrate that half of the patients have reduced exercise capacity, yet, only a minority of patients have pulmonary limitation. Thus, CPET is a helpful tool to further dissect reduced exercise tolerance and interpret exertional dyspnoea. With similar previous findings in survivors of SARS, physical deconditioning seems to be the main cause of impaired exercise capacity after severe coronavirus infections, and might even represent the natural course after severe lung injury with critical illness in general.

In summary, we demonstrate that physical deconditioning is the most common cause of impaired $V_{\mathrm{O}_{2} \max }$ in patients after severe COVID-19 pneumonitis. Whether these findings are specific to SARS-CoV-2 infection or conversely depict the common sequelae after ARDS caused by any insult has to be further explored. Finally, our findings underscore the importance of an early rehabilitative intervention in survivors of severe COVID-19 pneumonitis.

Kathleen Jahn ${ }^{1}$, Mihaela Sava ${ }^{2}$, Gregor Sommer $\oplus^{3}$, Desiree M. Schumann ${ }^{1}$, Stefano Bassetti ${ }^{4}$, Martin Siegemund ${ }^{5,6}$, Manuel Battegay ${ }^{2}$, Daiana Stolz ${ }^{1}$, Michael Tamm ${ }^{1}$, Nina Khanna ${ }^{2}$ and Katrin E. Hostettler ${ }^{1}$

${ }^{1}$ Clinics of Respiratory Medicine, University Hospital Basel and University of Basel, Basel, Switzerland. ${ }^{2}$ Division of Infectious Diseases and Hospital Epidemiology, University Hospital Basel and University of Basel, Basel, Switzerland. ${ }^{3}$ Dept of Radiology, University Hospital Basel and University of Basel, Basel, Switzerland. ${ }^{4}$ Division of Internal Medicine, University Hospital Basel and University of Basel, Basel, Switzerland. ${ }^{5}$ Dept of Clinical Research, University Hospital Basel and University of Basel, Basel, Switzerland. ${ }^{6}$ Dept of Intensive Care Medicine, University Hospital Basel and University of Basel, Basel, Switzerland.

Corresponding author: Katrin E. Hostettler (katrin.hostettler@usb.ch)

Conflict of interest: S. Bassetti participated on an advisory board for Pharming Technologies BV, outside the submitted work. All other authors have nothing to disclose.

\section{References}

$1 \quad$ Li X, Zeng W, Li X, et al. CT imaging changes of coronavirus disease 2019 (COVID-19): a multi-center study in Southwest China. J Transl Med 2020; 18: 154.

2 Huang $\mathrm{Y}$, Tan C, Wu J, et al. Impact of coronavirus disease 2019 on pulmonary function in early convalescence phase. Respir Res 2020; 21: 163.

3 Guler SA, Ebner L, Beigelman C, et al. Pulmonary function and radiological features 4 months after COVID-19: first results from the national prospective observational Swiss COVID-19 lung study. Eur Respir J 2021; 57: 2003690.

4 Sava M, Sommer G, Daikeler T, et al. Ninety-day outcome of patients with severe COVID-19 treated with tocilizumab - a single centre cohort study. Swiss Med Wkly 2021; 151: w20550.

5 Palange P, Ward SA, Carlsen KH, et al. Recommendations on the use of exercise testing in clinical practice. Eur Respir J 2007; 29: 185-209.

6 Borg G. Psychophysical scaling with applications in physical work and the perception of exertion. Scand J Work Environ Health 1990; 16: Suppl. 1, 55-58.

7 Wasserman K, D Sue JH, Stringer W, et al. Principles of Exercise Testing and Interpretation. Philadelphia, Lippincott Williams \& Wilkins, 2004; p. 612.

8 Schmid A, Schilter D, Fengels I, et al. Design and validation of an interpretative strategy for cardiopulmonary exercise tests. Respirology 2007; 12: 916-923.

9 Sonnweber T, Sahanic S, Pizzini A, et al. Cardiopulmonary recovery after COVID-19: an observational prospective multicentre trial. Eur Respir J 2021; 57: 2003481.

10 González J, Benítez ID, Carmona P, et al. Pulmonary function and radiological features in survivors of critical COVID-19: a 3-month prospective cohort. Chest 2021; 160: 187-198.

11 Mo X, Jian W, Su Z, et al. Abnormal pulmonary function in COVID-19 patients at time of hospital discharge. Eur Respir J 2020; 55: 2001217.

12 Paneroni M, Simonelli C, Saleri M, et al. Muscle strength and physical performance in patients without previous disabilities recovering from COVID-19 pneumonia. Am J Phys Med Rehabil 2021; 100: 105-109. 
13 Rinaldo RF, Mondoni M, Parazzini EM, et al. Deconditioning as main mechanism of impaired exercise response in COVID-19 survivors. Eur Respir J 2021; 58: 2100870.

14 Singh I, Joseph P, Heerdt PM, et al. Persistent exertional intolerance after COVID-19: insights from invasive cardiopulmonary exercise testing. Chest 2021; in press [https://doi.org/10.1016/j.chest.2021.08.010].

15 Skjørten I, Ankerstjerne OAW, Trebinjac D, et al. Cardiopulmonary exercise capacity and limitations 3 months after COVID-19 hospitalisation. Eur Respir J 2021; 58: 2100996.

16 Barbagelata L, Masson W, Iglesias D, et al. Cardiopulmonary exercise testing in patients with post-COVID-19 syndrome. Med Clin (Barc) 2021; in press [https://doi.org/10.1016/j.medcli.2021.07.007].

17 Clavario P, De Marzo V, Lotti R, et al. Cardiopulmonary exercise testing in COVID-19 patients at 3 months follow-up. Int J Cariol 2021; 340: 113-118.

18 Alba GA, Ziehr DR, Rouvina JN, et al. Exercise performance in patients with post-acute sequelae of SARS-CoV-2 infection compared to patients with unexplained dyspnea. EClinicalMedicine 2021; 39: 101066.

19 McGonagle D, O'Donnell JS, Sharif K, et al. Immune mechanisms of pulmonary intravascular coagulopathy in COVID-19 pneumonia. Lancet Rheumatol 2020; 2: e437-e445.

20 Gao Y, Chen R, Geng Q, et al. Cardiopulmonary exercise testing might be helpful for interpretation of impaired pulmonary function in recovered COVID-19 patients. Eur Respir J 2021; 57: 2004265.

21 Ong KC, Ng AW, Lee LS, et al. Pulmonary function and exercise capacity in survivors of severe acute respiratory syndrome. Eur Respir J 2004; 24: 436-442.

22 Neff TA, Stocker R, Frey HR, et al. Long-term assessment of lung function in survivors of severe ARDS. Chest 2003; 123: 845-853. 